\title{
Deep molecular response on imatinib treatment - results from a real-life retrospective study
}

\author{
Aleksandra Gołos ${ }^{1 *}$ (D), Joanna Niesiobędzka-Krężel ${ }^{2}$, Joanna Góra-Tybor ${ }^{3}$ iD \\ ${ }^{1}$ Department of Hematooncology, Comprehensive Cancer Center and Traumatology, Copernicus Memorial Hospital in Lodz, \\ Lodz, Poland
}

${ }^{2}$ Department of Hematology, Oncology and Internal Medicine, Medical University of Warsaw, Warsaw, Poland

${ }^{3}$ Department of Hematology, Medical University of Lodz, Lodz, Poland

\begin{abstract}
Introduction: Tyrosine kinase inhibitors (TKIs) have dramatically changed the outcome of chronic myeloid leukemia (CML) patients. Recent research focused on TKI discontinuation after achieving a deep molecular response (DMR) has revealed that about half of the patients maintain the response. DMR is a key criterion for TKI discontinuation. Our retrospective, 'real-life' study was aimed at to estimating the proportion of patients treated with first-line imatinib (IM) who achieved DMR and thus may be candidates for discontinuation of TKI treatment in a real life setting.
\end{abstract}

Material and methods: Two hundred and twenty-three patients were enrolled. All patients started IM at $400 \mathrm{mg}$ daily. The median age at the time of diagnosis was 57 years (range: 17-92).

Results: Eighty-five patients (43\%) in the whole group achieved DMR. Early molecular response (EMR) was achieved by $136(69 \%)$ patients and correlated with the DMR rate (53\% with EMR vs. $14 \%$ without, $p<0.001)$. Major molecular response (MMR) after a year of treatment was confirmed in 108 (55\%) patients, and was predictive for achieving DMR at any time (69\% with MMR vs. $24 \%$ without, $p<0.001)$.

Conclusion: DMR can be achieved in a significant proportion of patients in a real-life setting. We observed that both the achievement of an EMR at three months and MMR at 12 months were associated with a significant advantage in terms of DMR.

Key words: chronic myeloid leukemia, imatinib, deep molecular response

Acta Haematologica Polonica 2021; 52, 5: 504-508

\section{Introduction}

Chronic myeloid leukemia (CML) is the first myeloid neoplasm in which targeted therapy has been used. BCR-ABL kinase inhibitors (TKIs) have dramatically changed the outcome of CML patients, prolonging median survival to nearly normal life expectancy [1, 2]. The first TKI introduced for therapy was imatinib (IM). Over the following years, second generation TKIs of greater potency, namely nilotinib, dasatinib, and bosutinib, were introduced [3]. Over the time since TKls were first used, the goals of treatment have evolved and become more stringent in optimizing treatment strategy. At present, the definition of optimal responses according to European LeukemiaNet (ELN) recommendations is: to achieve early molecular response (EMR); this is defined as $B C R-A B L 1$ transcript reduction $\leq 10 \%$ at three months, $\leq 1 \%$

\footnotetext{
*Address for correspondence: Aleksandra Gołos, Department of Hematooncology, Comprehensive Cancer Center and Traumatology, Copernicus Memorial Hospital in Lodz, Pabianicka 62, 93-513 Lodz, Poland, phone +48 4268954 54, fax +48 4268951 92, e-mail: alexandra_golos@tlen.pl
}
PTHiT Copyright (c 2021 The Polish Society of Haematologists and Transfusiologists, Insitute of Haematology and Transfusion Medicine. All rights reserved.


or complete cytogenetics response (CCyR) at six months, $\leq 0.1 \%$ at 12 months, and $\leq 0.01 \%$ at 24 months [4].

Several retrospective studies have shown that EMR at three months predicts significantly better long-term outcomes: event-free survival (EFS), progression-free survival (PFS), and overall survival (OS), and this is the case both in patients treated with IM and in patients treated with second-generation TKIs [1, 4]. Beyond EMR, two additional molecular responses have been defined as a prognostic factor for a long-term outcome: major molecular response (MMR: BCR-ABL <0.1\%), and deep molecular response (DMR), defined as BCR-ABL $\leq 0.01 \%$. The achievement of DMR is per se an important prognostic factor for the long-term clinical outcome [5].

Recently, the possibility of discontinuing TKI treatment in CML patients with a deep molecular response (DMR) has been explored in clinical trials [6, 7]. Their results have indicated that about half of the patients maintain the response. Considering the potential side effects of lifelong exposure to TKIs, achieving treatment-free remission (TFR) has become a key goal of CML therapy [8].

Our study aimed to estimate the proportion of patients treated with first-line IM who achieve DMR and thus may be candidates for the discontinuation of TKI treatment. Additionally, we analyzed adherence to the recommendations according to monitoring cytogenetic and molecular response in a real-life setting. Furthermore, various clinical and laboratory parameters were investigated as potential predictors of DMR.

\section{Material and methods}

This retrospective analysis included newly diagnosed patients with chronic phase $\mathrm{CML}$ who received IM upfront in two Polish hematological centers between 2013 and 2018 outside of clinical trials. All living patients have given their written consent and the study was approved by the local ethics committee. Diagnostic criteria were defined according to the ELN criteria 2013 [9]. Sokal, Hasford, and EUTOS scores were used to stratify risk at the time of diagnosis [10]. Molecular responses were assessed according to standard criteria and presented as International Scale (IS): MMR $-B C R-A B L^{\text {Is }}<0.1 \%, M^{4.0}-B C R-A B L^{\text {is }}<0.01 \%$, and $M R^{4.5}-B C R-A B L^{\text {Is }}<0.001 \%$ [9]. DMR was defined as an achievement of at least $\mathrm{MR}^{4.0}$ or deeper. EMR was defined as $B C R-A B L$ transcript reduction $<10 \%$ after three months of IM treatment [9]. Cytogenetics analyses were performed on at least 20 marrow metaphases. CCyR was defined according to the ELN recommendations 2013 [9]. The time points of response assessments were as follows: six and 12 months of IM treatment for a cytogenetic response, and three, six, nine, and 12 months for a molecular response.

We used univariate logistic regression to estimate the impact of selected factors on the response. For each variable, the odds ratio and 95\% confidence interval (95\% $\mathrm{Cl})$ were calculated. Percentages were compared using the chi-squared test. All performed tests were two-sided, and results were considered statistically significant if $p<0.05$. Stata 15 was used for statistical analyses.

\section{Results}

\section{Patient characteristics}

Two hundred and twenty-three patients who were diagnosed with CML - CP between 2013 and 2018 were enrolled in the study. All patients received IM at $400 \mathrm{mg}$ daily. The median age at the time of diagnosis was 57 years (range: $17-92$, with 36 [16\%] patients $>70$ years old). All patients had chromosome Philadelphia in the baseline cytogenetics. According to the risk scores, the majority of patients were low risk (79\% by Sokal, $71 \%$ by Hasford, and $89 \%$ by EUTOS). Median follow-up was 55 months (range: 12-100). The baseline characteristics of the group are set out in Table I.

\section{Imatinib tolerance}

IM was well tolerated. Adverse events (AEs) of any grade occurred in 63 (28\%) patients. No cardiovascular event occurred. Imatinib was reduced to $300 \mathrm{mg}$ in 32 patients (14\%). The most common AEs are listed in Table II.

\section{Change for second-line treatment}

Overall, 81 patients (36\%) discontinued imatinib and switched to a second-generation TKI. Among these, 66 (81\%) were refractory to imatinib, eight (10\%) switched due to imatinib intolerance, and seven $(9 \%)$ for both reasons. The median time to switch was 12 months (range: 3-84). Nilotinib and dasatinib were chosen as second-line therapy with almost equal frequency (49\% and 48\%, respectively), while only two patients (3\%) switched to bosutinib. During the follow-up, four patients progressed to advanced phases of CML [three patients to accelerated phase (AP) and one to blast phase (BP)].

\section{Response evaluation}

Optimal response at particular time points was assessed according to the ELN 2013 [9]. The proportions of patients who had been tested are shown in Table III. Almost all of the patients had cytogenetics and molecular analysis after six months of treatment (95\%). Response assessment after 12 months of imatinib treatment was done by cytogenetics and molecular methods in 190 (85\%), and 196 (87\%) patients, respectively.

\section{Response to IM therapy}

The optimal responses to imatinib according to the ELN 2013 criteria are presented in Table IV. The median time to achieve the deepest response was 12 months (range: 3-90). 
Table I. Patient characteristics

\begin{tabular}{|c|c|}
\hline Characteristics & $\mathbf{N}[\%]$ \\
\hline Number of patients & 223 \\
\hline Gender & \\
\hline Female & $91(41)$ \\
\hline Male & $132(59)$ \\
\hline Median age at diagnosis (range) & $57(17-92)$ \\
\hline $\mathrm{Ph}+$ clone & \\
\hline With CCA & $19(8)$ \\
\hline Variant & $8(3)$ \\
\hline Type of transcript & \\
\hline P210 & $221(99)$ \\
\hline $\mathrm{e} 14 \mathrm{a} 2$ & $144(65)$ \\
\hline e13a2 & $75(34)$ \\
\hline e13a3 & $1(0.5)$ \\
\hline e14a3 & $1(0.5)$ \\
\hline P190 & $1(0,5)$ \\
\hline $\mathrm{e} 1 \mathrm{a} 2$ & $1(0.5)$ \\
\hline P230 & $1(0,5)$ \\
\hline e19a2 & $1(0.5)$ \\
\hline Sokal score (total) & 221 \\
\hline Low & $174(79)$ \\
\hline Intermediate & $36(16)$ \\
\hline High & $11(5)$ \\
\hline Hasford score (total) & 221 \\
\hline Low & $156(71)$ \\
\hline Intermediate & $51(23)$ \\
\hline High & $14(6)$ \\
\hline EUTOS score (total) & 221 \\
\hline Low & $198(89)$ \\
\hline High & $23(11)$ \\
\hline
\end{tabular}

$\mathrm{Ph}$ - Philadelphia; CCA - clonal cytogenetics abnormalities

At three months, 136 patients (69\%) obtained EMR. In this group, 72 patients (53\%) achieved DMR. The percentage of DMR in the group without optimal EMR was 14\% (95\% Cl: $0.24-0.45, p<0.001)$. After a year of treatment, MMR was confirmed in 108 patients (55\%). In this subgroup, 75 patients (69\%) achieved DMR at any time. In contrast, only 21 (24\%) patients without MMR after one year achieved DMR (95\% Cl: 0.43-0.65, p <0.001). In general, during IM treatment 85 patients (43\%) in the whole group achieved DMR.

The percentage of patients with treatment failure at a particular time point, according to the ELN 2013 recommendations, are presented in Table V. In general, during imatinib treatment, 49 patients (22\%) did not achieve MMR, and 25 (11\%) did not reach PCyR. These patients were considered as refractory to imatinib and switched to second-generation TKI except for 10 patients (4\%) who were still treated with imatinib because of their advanced age and comorbidities. They were considered as having
Table II. Main side effects of imatinib

\begin{tabular}{|l|c|c|}
\hline Side effect of imatinib & \multicolumn{1}{|c|}{$\begin{array}{c}\text { Number } \\
\text { of patients } \\
\text { with side effect } \\
\text { at any grade }\end{array}$} & $\begin{array}{c}\text { Number } \\
\text { of patients } \\
\text { with grade 4 } \\
\text { toxicity, N [\%] }\end{array}$ \\
\cline { 2 - 3 } & $63(28 \%)$ & $15(7)$ \\
\hline Hematological toxicity & $7(11 \%)$ & \\
\hline Thrombocytopenia & $3(4 \%)$ & $4(27)$ \\
\hline Anemia & $6(10 \%)$ & $1(6)$ \\
\hline Neutropenia & $4(27)$ \\
\hline Non-hematological toxicity & $13(20 \%)$ & 0 \\
\hline Edema & $10(16 \%)$ & 0 \\
Nausea, vomiting & $4(6 \%)$ & $2(13)$ \\
Hepatic toxicity & $4(6 \%)$ & $1(7)$ \\
Renal impairment & $7(11 \%)$ & $3(20)$ \\
Skin rash & $19(30 \%)$ & 0 \\
Bone/muscle pain & $22(35 \%)$ & 0 \\
Other & &
\end{tabular}

Table III. Number of patients screened at designated time points

\begin{tabular}{|l|c|c|}
\hline $\begin{array}{l}\text { Method of } \\
\text { evaluation }\end{array}$ & $\begin{array}{c}\text { Time point } \\
\text { of evaluation }\end{array}$ & $\begin{array}{c}\text { Number of screened } \\
\text { patients [\%] }\end{array}$ \\
\hline Cytogenetics & $6^{\text {th }}$ month & $213(95)$ \\
& $12^{\text {th }}$ month & $190(85)$ \\
\hline Molecular & $3^{\text {rd }}$ month & $197(88)$ \\
tests & $6^{\text {th }}$ month & $214(95)$ \\
& $9^{\text {th }}$ month & $152(68)$ \\
& $12^{\text {th }}$ month & $196(87)$ \\
\hline
\end{tabular}

Table IV. Number of patients with optimal response according to ELN 2013 criteria

\begin{tabular}{l|c}
\hline $\begin{array}{l}\text { Optimal responses according to } \\
\text { ELN recommendations }\end{array}$ & Total (\%) \\
\hline CCyR at $6^{\text {th }}$ month & $151(67.7)$ \\
CCyR at $12^{\text {th }}$ month & $157(70)$ \\
$M M R$ at $12^{\text {th }}$ month & $108(48.4)$ \\
$>\mathrm{MR}^{4.0}$ at any time point & $47(21)$ \\
$\geq \mathrm{MR}^{4.5}$ at any time point & $48(22 \%)$
\end{tabular}

CCyR - complete cytogenetic response; ELN - European LeukemiaNet; MMR - major molecular response; MR - molecular response

clinical benefit although without an optimal response. Over the follow-up, four patients progressed to advanced phases of CML: three patients to AP and one to BP. All of the progressions occurred on imatinib.

Among 36 patients older than 70 years at the time of diagnosis, 50\% achieved EMR, 33\% MMR after a year of treatment, and 30\% have ever achieved DMR. 
Table V. Number of patients with treatment failure according to ELN 2013 criteria

\begin{tabular}{l|c}
\hline $\begin{array}{l}\text { Treatment failure according to ELN } \\
\text { recommendations }\end{array}$ & Total [\%] \\
\hline No CHR at 3 months & $1(0.5)$ \\
No MCYR at 6 month & $19(9)$ \\
$B C R-A B L>10 \%$ at 6 month & $32(14)$ \\
No CCYR at 12 month & $31(14)$ \\
$B C R-A B L>1 \%$ at 12 month & $30(13)$ \\
\hline
\end{tabular}

CCyR - complete cytogenetic response; CHR - complete hematologic response; ELN - European LeukemiaNet; MCyR - major cytogenetic response

We analyzed the impact of age, Sokal, Hasford, and EUTOS scores, and the achievement of EMR on response. Additionally, we analyzed whether the presence of clonal cytogenetic abnormalities (CCA) influenced cytogenetic responses at six and 12 months, and molecular response at three, six, and $12^{\text {th }}$ months. In univariate analysis, only the achievement of EMR at three months predicted the chance of DMR [OR 5.88 (95\% Cl: 2.75-12.59), p <0.001). Other than that, a low Hasford score was associated with the probability of EMR achievement [OR 2.03 (95\% Cl: 1.04-3.97), $p=0.03$ ]. Other factors did not impact upon molecular or cytogenetic response at any time point.

\section{Discussion}

The main criterion for safe and appropriate TKI cessation is the achievement of DMR, defined as BCR-ABL1 $\leq 0.01 \%$ $[3,9]$. Therefore, in routine clinical practice, it is crucial to monitor molecular response at designated time points following the ELN recommendations [9].

The results of our study reveal that in a 'real-life' setting, compliance with monitoring recommendations is very good. Nearly $90 \%$ of patients had both molecular and cytogenetic testing in the proper time points. In contrast, Nicolinii et al. reported that among $418 \mathrm{CML}$ patients included in a real-life retrospective study in France, molecular data was fully available for 294 patients (70\%) [10]. In a real-life Italian study, the EMR data was available only in $61 \%$ of patients [11].

It has been shown that EMR is a strong predictor of outcome of CML patients regarding both PFS and OS [5]. Moreover, the achievement of DMR is influenced by the molecular response at three months of therapy. Marin et al. identified BCR-ABL of $0.61 \%$ at three months to be a discriminative landmark for the achievement of cumulative complete molecular remission with a difference of $85 \%$ vs. $2 \%$ at 8 years [12]. In our study, $69 \%$ of patients achieved optimal EMR. Among them, 53\% achieved DMR at any time, whereas the percentage of DMR in the group without optimal EMR was only $14 \%(p<0.001)$ which confirms the importance of a 3-month molecular response for
DMR achievement. In two real-life Italian studies, the percentage of optimal EMR on IM was similar and amounted to around $80 \%[11,13]$.

In our group, $55 \%$ of patients achieved MMR at 12 months. This is another important milestone in $\mathrm{CML}$ treatment, significant both for DMR and OS [5]. Sixty-nine percent of patients with MMR at 12 months achieved DMR later on. In contrast, only $24 \%$ of patients who did not have MMR at 12 months achieved DMR $(p<0.001)$ at any time during imatinib treatment. In line with our results, Molica et al. observed MMR at 12 months in $57.1 \%$ of patients [13]. They underscored that among patients who had not achieved MMR at 12 months, 42.7\% never reached MMR during IM treatment.

We have reported that $43 \%$ of patients achieved DMR $\left(\mathrm{MR}^{4.0} 21 \%, \geq \mathrm{MR}^{4.5} 22 \%\right)$ after five years of follow up. Our results are in line with previous real-life studies [8, 9]. Nevertheless, in the randomized German CML - Study IV, a significantly higher proportion of patients achieved DMR [1]. At 10 years, $59 \%$ of patients achieved $\mathrm{MR}^{5.0}, 72 \% \mathrm{MR}^{4.5}$, and $81 \% \mathrm{MR}^{4.0}$. However, it should be noted that the dose of IM was $800 \mathrm{mg}$ and the follow-up period was longer. In the randomized IRIS study, the response rate of $\mathrm{MR}^{4.5}$ after five years follow-up was $40 \%$, which was similar to our report, and after 10 years had increased to 63\% [2].

Patients in clinical trials are selected according to eligibility criteria. The exclusion criteria are mainly associated with co-morbidities. Such preselection results in a lower age of the studied population. In our study, 16\% of patients were $>70$ years old. Although univariate analysis showed no relationship between age and DMR, the frequency of EMR and DMR was significantly lower in patients $>70$ years old. This may have been associated with comorbidities, drug interactions, and the need to reduce the TKI dose due to toxicity. Despite this, $30 \%$ of older patients still achieved DMR.

\section{Conclusion}

Our study has shown that DMR can be achieved in a significant proportion of CML patients treated with IM as first-line therapy in a 'real-life' setting. We observed that both the achievement of an EMR at three months and MMR at 12 months were associated with a significant advantage in terms of DMR.

Therefore, if these therapeutic goals are not achieved, for selected patients switching to a strong-acting second-generation TKI should be considered.

\section{References}

1. Kalmanti L, Saussele S, Lauseker M, et al. Safety and efficacy of imatinib in CML over a period of 10 years: data from the randomized CML-study IV. Leukemia. 2015; 29(5): 1123-1132, doi: 10.1038/ leu.2015.36, indexed in Pubmed: 25676422. 
2. Hochhaus A, Larson R, Guilhot F, et al. Long-term outcomes of imatinib treatment for chronic myeloid leukemia. N Engl J Med. 2017; 376(10): 917-927, doi: 10.1056/nejmoa1609324.

3. Jabbour E, Kantarjian HM, O'Brien S, et al. Front-line therapy with second-generation tyrosine kinase inhibitors in patients with early chronic phase chronic myeloid leukemia: what is the optimal response? J Clin Oncol. 2011; 29(32): 4260-4265, doi: 10.1200/ JC0.2011.36.0693, indexed in Pubmed: 21990394.

4. Hochhaus A, Baccarani M, Silver RT, et al. European LeukemiaNet 2020 recommendations for treating chronic myeloid leukemia. Leukemia. 2020; 34(4): 966-984, doi: 10.1038/s41375-020-0776-2, indexed in Pubmed: 32127639.

5. Jain P, Kantarjian H, Nazha A, et al. Early responses predict better outcomes in patients with newly diagnosed chronic myeloid leukemia: results with four tyrosine kinase inhibitor modalities. Blood. 2013; 121(24): 4867-4874, doi: 10.1182/blood-2013-03-490128, indexed in Pubmed: 23620574.

6. Etienne G, Guilhot J, Rea D, et al. Long-term follow-up of the french stop imatinib (STIM1) study in patients with chronic myeloid leukemia. J Clin Oncol. 2017; 35(3): 298-305, doi: 10.1200/JC0.2016.68.2914, indexed in Pubmed: 28095277.

7. Saussele S, Richter J, Guilhot J, et al. EURO-SKI investigators. Discontinuation of tyrosine kinase inhibitor therapy in chronic myeloid leukaemia (EURO-SKI): a prespecified interim analysis of a prospective, multicentre, non-randomised, trial. Lancet Oncol. 2018; 19(6): 747-757, doi: 10.1016/S1470-2045(18)30192-X, indexed in Pubmed: 29735299.
8. Hughes TP, Ross DM. Moving treatment-free remission into mainstream clinical practice in CML. Blood. 2016; 128(1): $17-$ -23, doi: 10.1182/blood-2016-01-694265, indexed in Pubmed: 27013442.

9. Baccarani M, Deininger MW, Rosti G, et al. European LeukemiaNet recommendations for the management of chronic myeloid leukemia: 2013. Blood. 2013; 122(6): 872-884, doi: 10.1182/ blood-2013-05-501569, indexed in Pubmed: 23803709.

10. Nicolini FE, Alcazer V, Cony-Makhoul P, et al. Long-term follow-up of de novo chronic phase chronic myelogenous leukemia patients on front-line imatinib. Exp Hematol. 2018; 64: 97-105. e4, doi: 10.1016/j.exphem.2018.05.003, indexed in Pubmed: 29800673.

11. Castagnetti F, Di Raimondo F, De Vivo A, et al. A population-based study of chronic myeloid leukemia patients treated with imatinib in first line. Am J Hematol. 2017; 92(1): 82-87, doi: 10.1002/ajh.24591, indexed in Pubmed: 27770583.

12. Marin D, Ibrahim AR, Lucas C, et al. Assessment of BCR-ABL1 transcript levels at 3 months is the only requirement for predicting outcome for patients with chronic myeloid leukemia treated with tyrosine kinase inhibitors. J Clin Oncol. 2012; 30(3): 232-238, doi: 10.1200/ JC0.2011.38.6565, indexed in Pubmed: 22067393.

13. Molica M, Colafigli G, Scalzulli E, et al. Ten-year outcome of chronic-phase chronic myeloid leukemia patients treated with imatinib in real life. Ann Hematol. 2019; 98(8): 1891-1904, doi: 10.1007/s00277019-03706-x, indexed in Pubmed: 31079264. 